\title{
Enhanced Chemical Immobilization and Catalytic Conversion of Polysulfide Intermediates Using Metallic Mo Nanoclusters for High-Performance Li-S Batteries
}

Yuanjian Li, ${ }^{\dagger}$ Chong Wang, ${ }^{\dagger}$ Wenyu Wang,${ }^{\dagger}$ Alex Yong Sheng Eng, ${ }^{\ddagger}$ Mintao Wan, ${ }^{\dagger}$ Lin Fu, ${ }^{\dagger}$ Eryang Mao,${ }^{\dagger}$ Guocheng Li,${ }^{\dagger}$ Jiang Tang,${ }^{\dagger}$ Zhi Wei Seh,,${ }^{\dagger}$ Yongming Sun ${ }^{\dagger *}$

$\dagger$ Wuhan National Laboratory for Optoelectronics, Huazhong University of Science and Technology, Wuhan 430074, China

$\$$ Institute of Materials Research and Engineering, A*STAR (Agency for Science, Technology and Research), 2 Fusionopolis Way, Singapore 138634, Singapore.

* Corresponding author.

E-mail address: yongmingsun@hust.edu.cn (Y. Sun). 

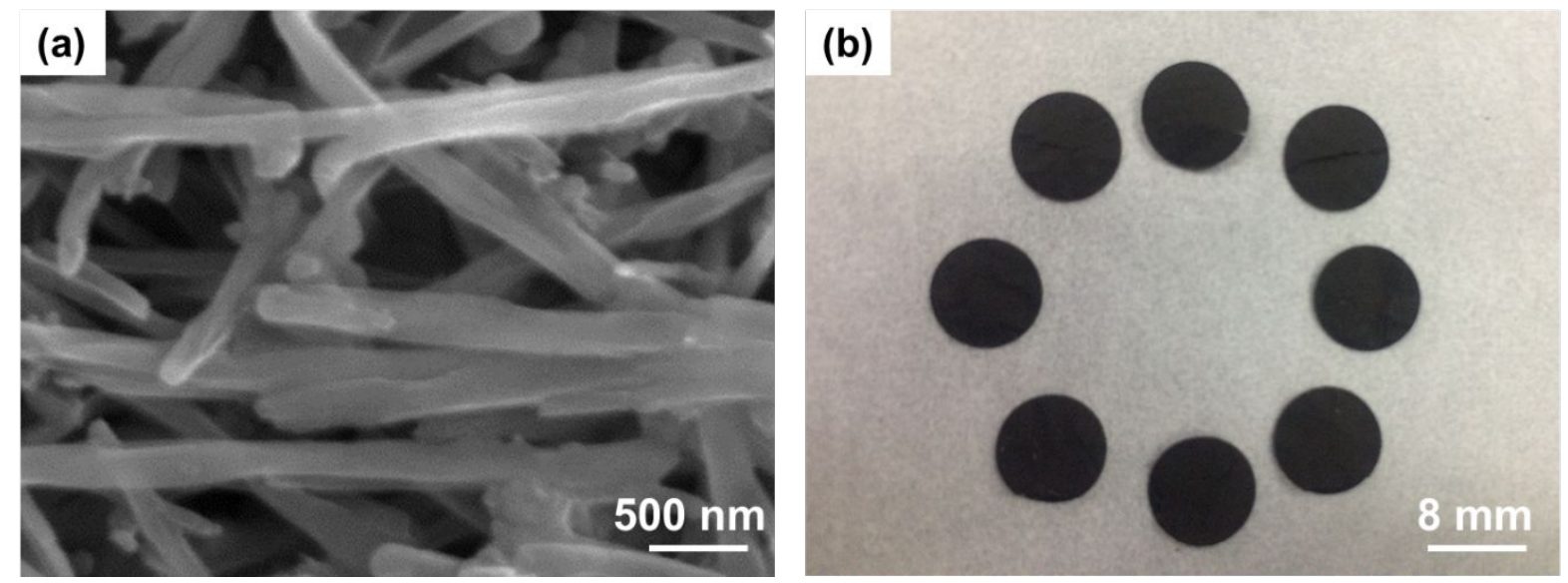

Figure S1. (a) SEM images and (b) digital photos of the pristine carbon nanofiber (CNF) substrates.

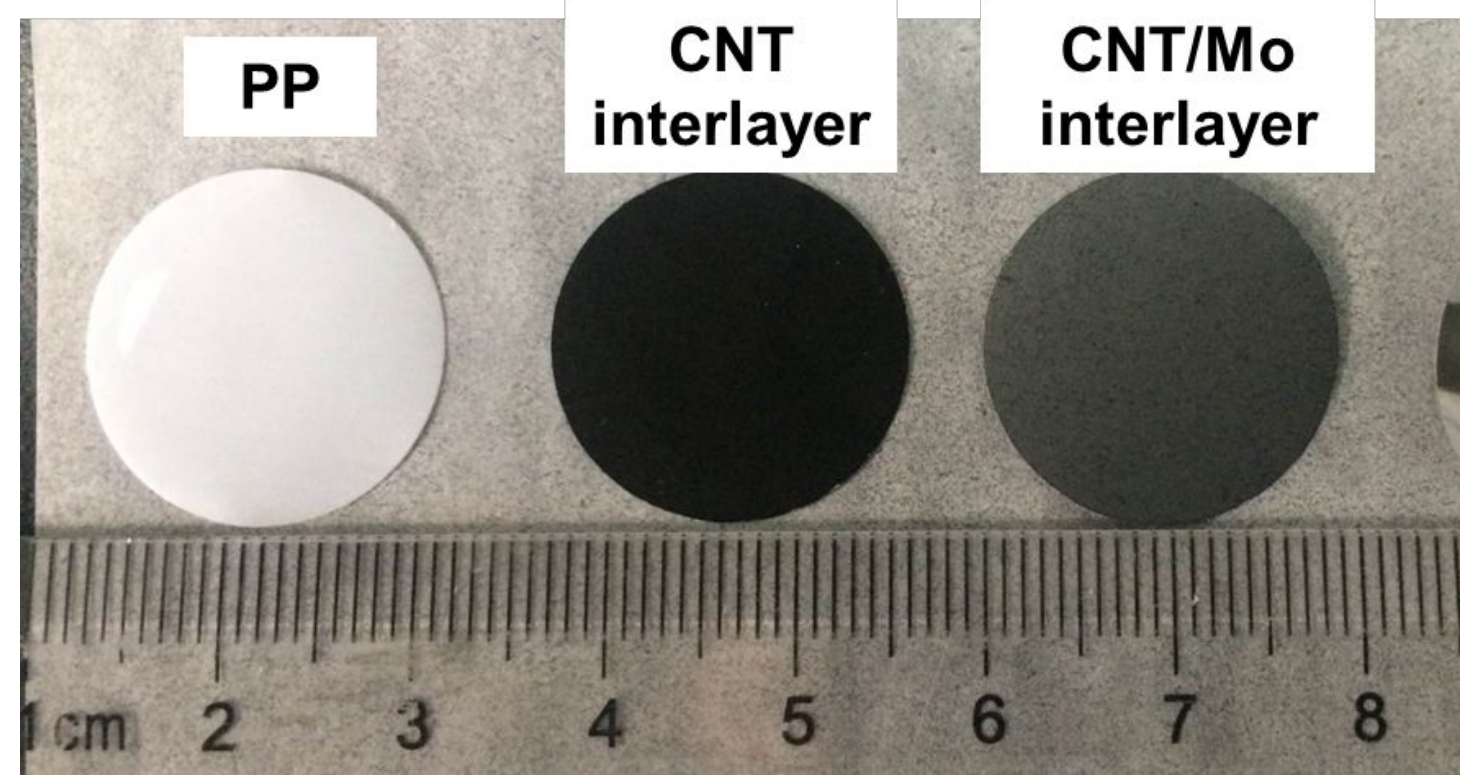

Figure S2. Digital photos of pristine polypropylene (PP) separator, carbon nanotube (CNT) film supported by PP separator and metallic Mo decorated CNT film supported by PP separator. 

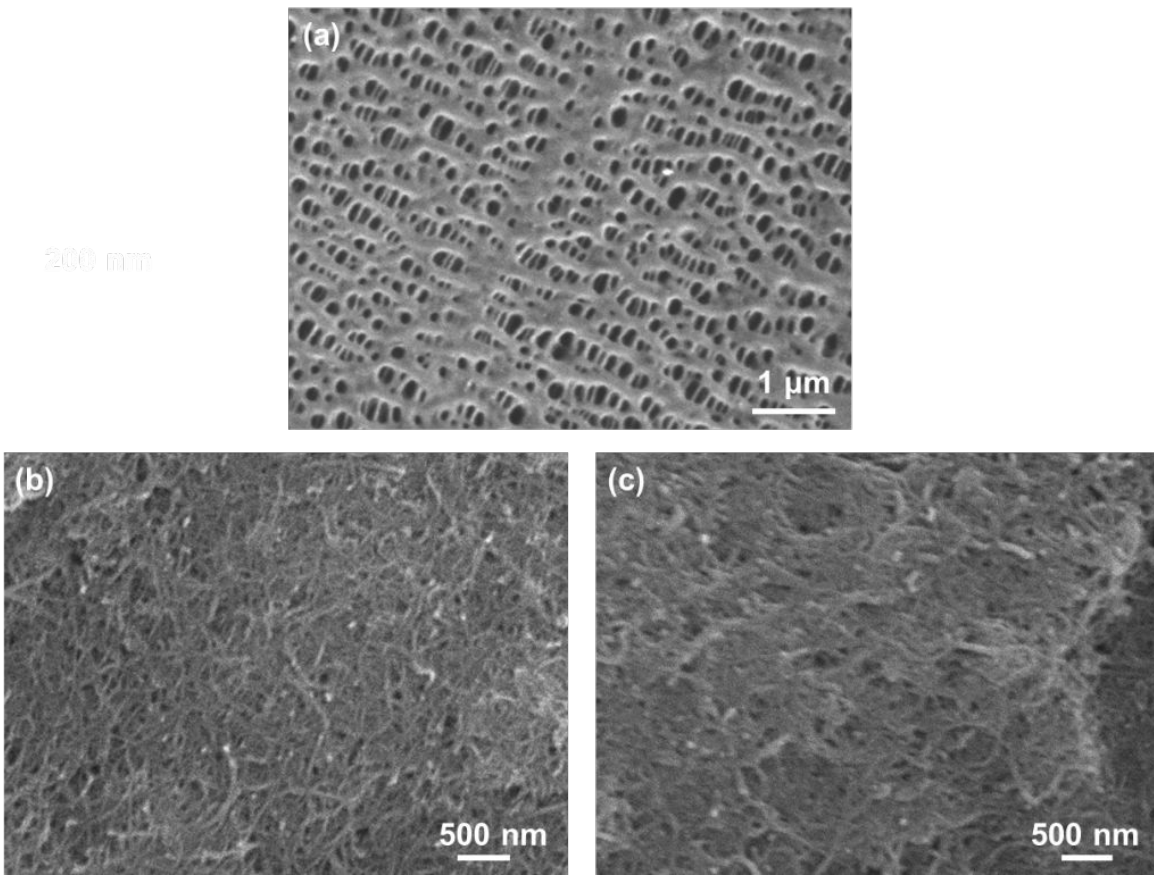

Figure S3. Top-view SEM images of (a) pristine PP separator, (b) CNT film and (c) CNT/Mo film, supported by PP separator.
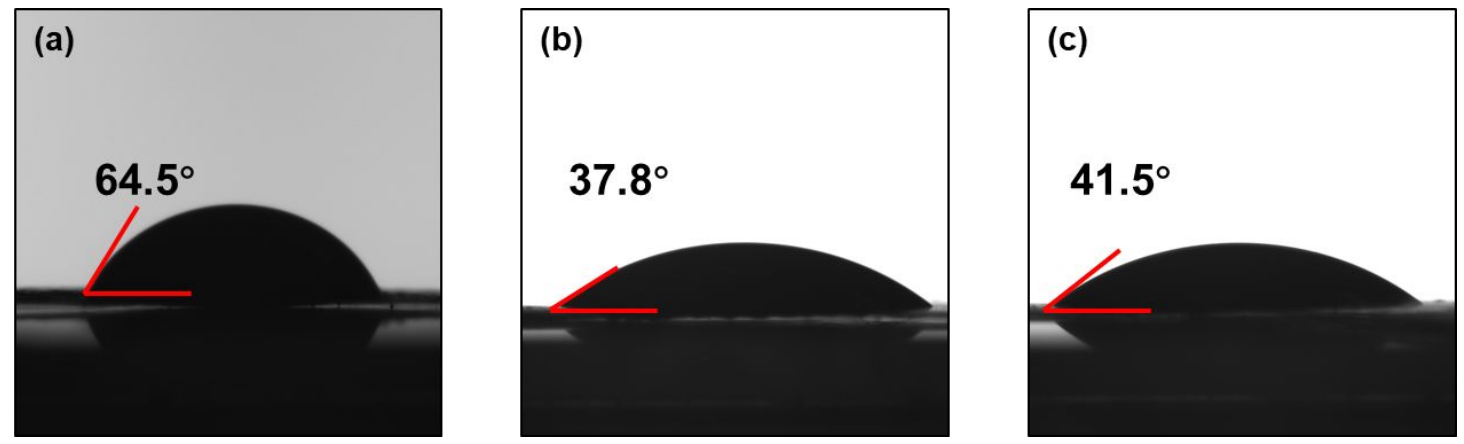

Figure S4. Contact angles between the electrolyte and (a) pristine PP separator and (b) CNT film and (c) CNT/Mo film, supported by PP separator. 


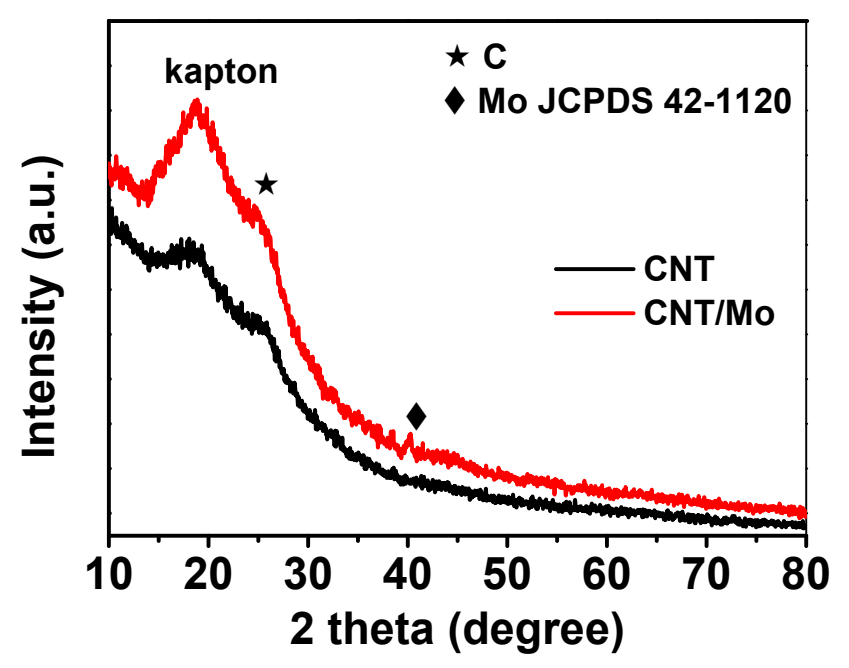

Figure S5. XRD patterns of CNT and CNT/Mo films.

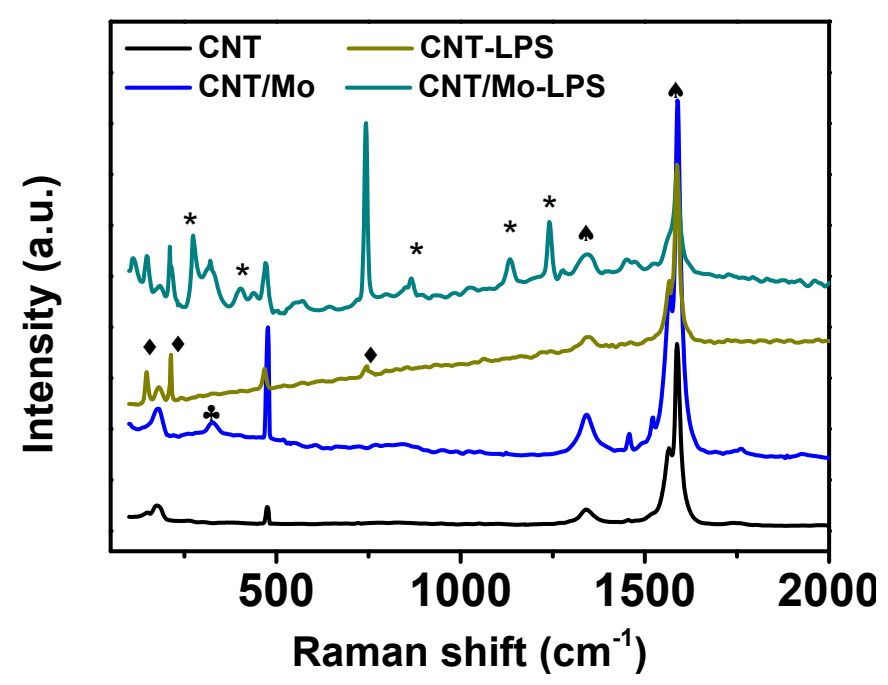

Figure S6. Raman spectra of CNT, CNT/Mo, CNT-LPS, and CNT/Mo-LPS composites.

All samples show peaks at 1340 (marked as $\mathbf{\text { s) }}$ and $1596 \mathrm{~cm}^{-1}$ (marked as $\boldsymbol{\vee}$ ) corresponding to the D band and G band of CNT (Figure S6). A new peak emerges at $180 \mathrm{~cm}^{-1}$ (marked with *) for the CNT/Mo in comparison to the pure CNT, which can be indexed to the $\mathrm{MoO}_{\mathrm{x}}$ species ${ }^{\mathrm{S} 1}$ and with XPS result (Figure 2j). New peaks are observed at 143, 218, and $750 \mathrm{~cm}^{-1}$ (marked with $\bullet$ ) in the Raman spectrum for the CNT- $\mathrm{Li}_{2} \mathrm{~S}_{6}$ composite in comparison to the pure CNT, which arise from polysulfides species. $^{\mathrm{S} 2}$, S3 Compared with the CNT/Mo and CNT-LPS composites, new peaks emerge in the range of 240 to $1250 \mathrm{~cm}^{-1}$ (marked with $*$ ), which are associated the formation of thiosulfate and polythionate species and other oxygen-containing inorganosulfur compounds, ${ }^{\mathrm{S} 4}$ which provides strong evidence of chemical interactions between the $\mathrm{MoO}_{\mathrm{x}}$ species and LPS. It is noted that no signal for $\mathrm{MoS}_{2}$ is observed using Raman analyses. The above Raman results are in good agreement with XPS analysis, and further confirmed the chemical interactions between the $\mathrm{MoO}_{\mathrm{x}}$ species 
and LPS

With PP separator
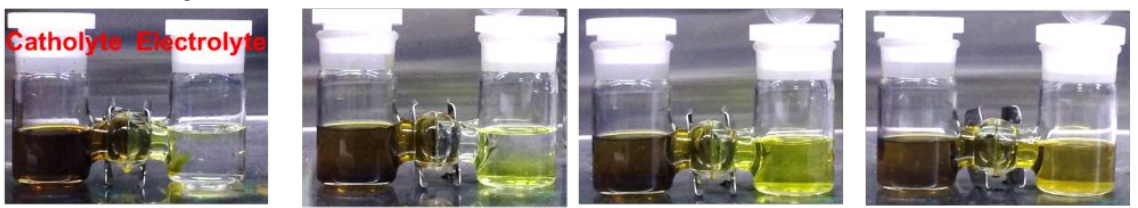

With CNT film
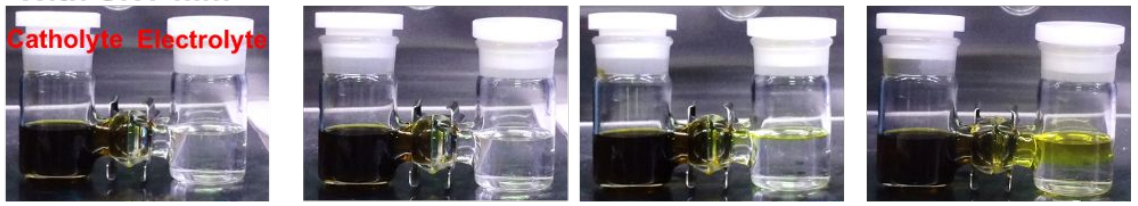

With CNT/Mo film
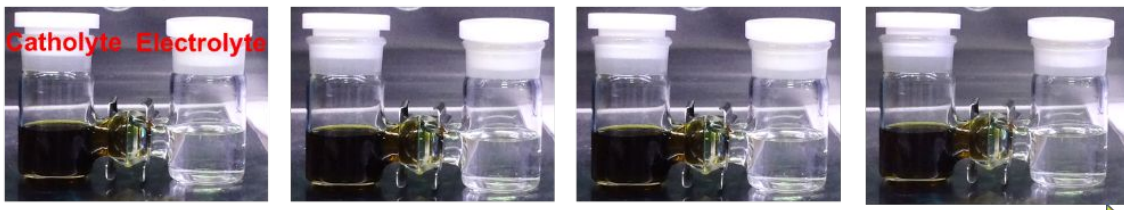

$5 \mathrm{~min}$

$6 h$

$12 \mathrm{~h}$

$24 \mathrm{~h}$

Figure S7. Optical images for the polysulfide diffusion test in glass cells with pure PP separator and PP separator with CNT, and CNT/Mo films, respectively.
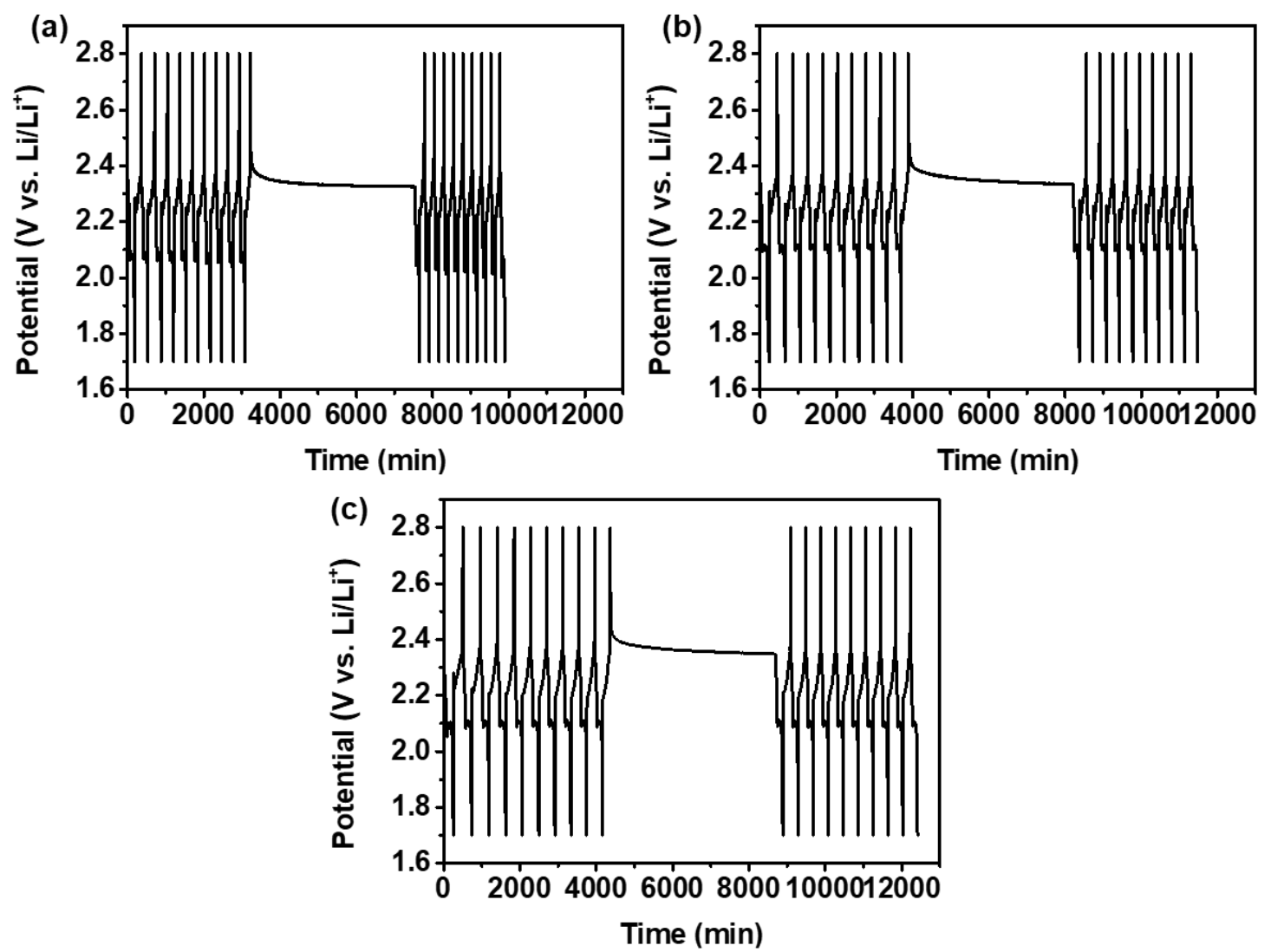

Figure S8. Potential-time curves during the interruption processes for Li-S cells with (a) $\mathrm{CNF} / \mathrm{LPS}$, (b) $\mathrm{CNF} / \mathrm{LPS} / \mathrm{CNT}$, and (c) CNT/LPS/Mo/CNT cathodes. Before the self-discharge measurement, the cells were pre-cycled for 10 times and rested for $72 \mathrm{~h}$ 
at the charge state.
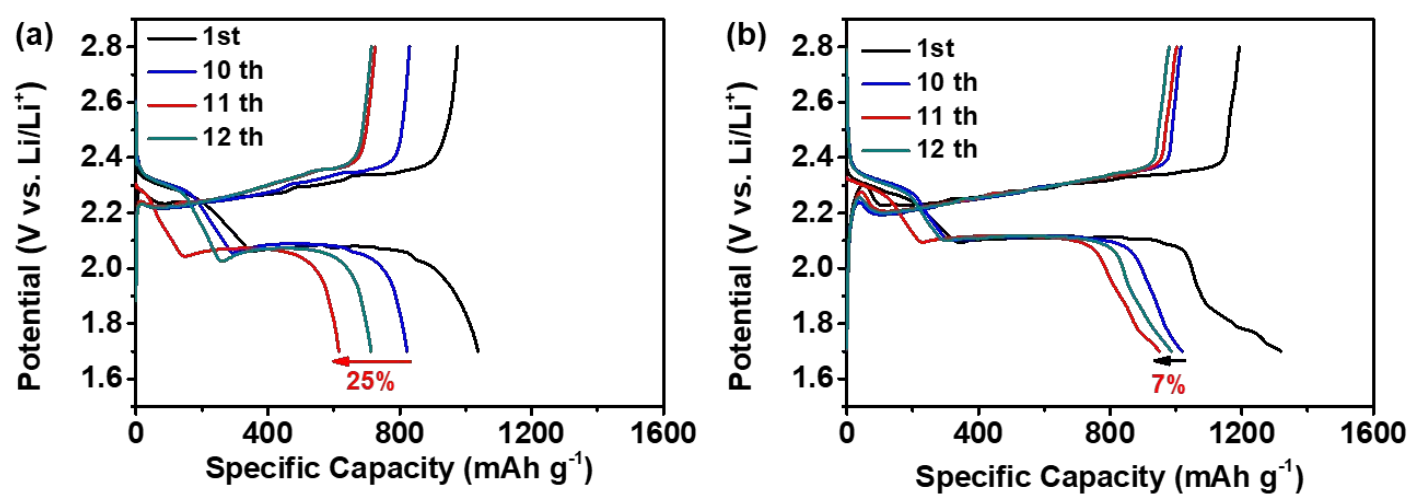

Figure S9. Representative charge-discharge voltage profiles upon the 10th, 11th and 12th cycle and average self-discharge of cells using (a) CNF/LPS and (b) $\mathrm{CNF} / \mathrm{LPS} / \mathrm{CNT}$ cathode.

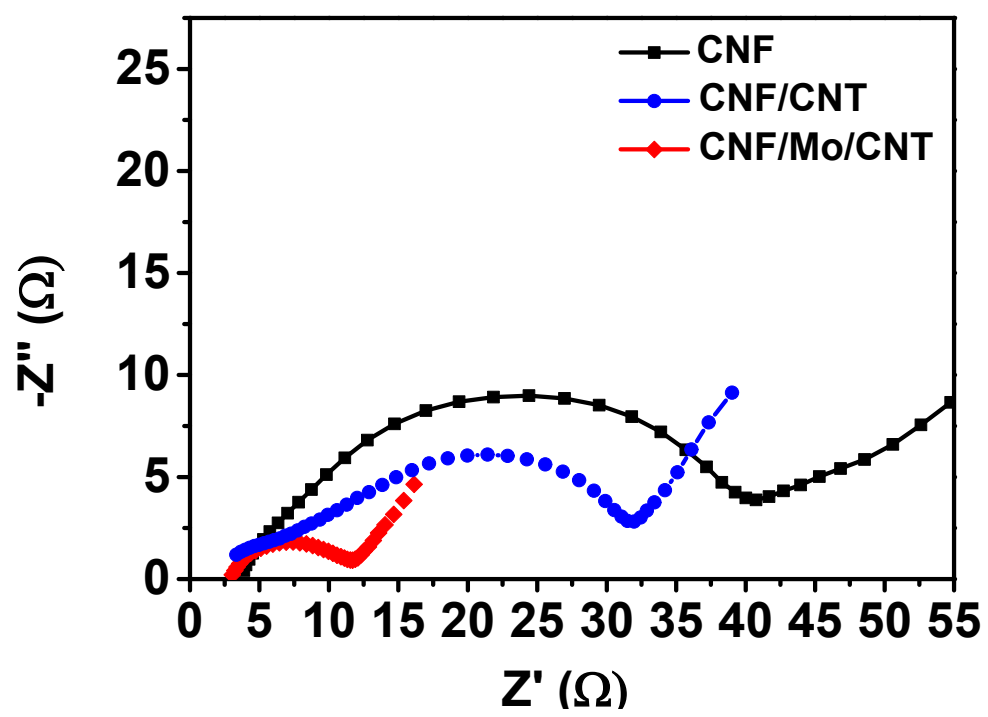

Figure S10. EIS spectra of the symmetrical cells with $\mathrm{CNF}, \mathrm{CNF} / \mathrm{CNT}$, and $\mathrm{CNF} / \mathrm{Mo} / \mathrm{CNT}$ electrodes. 

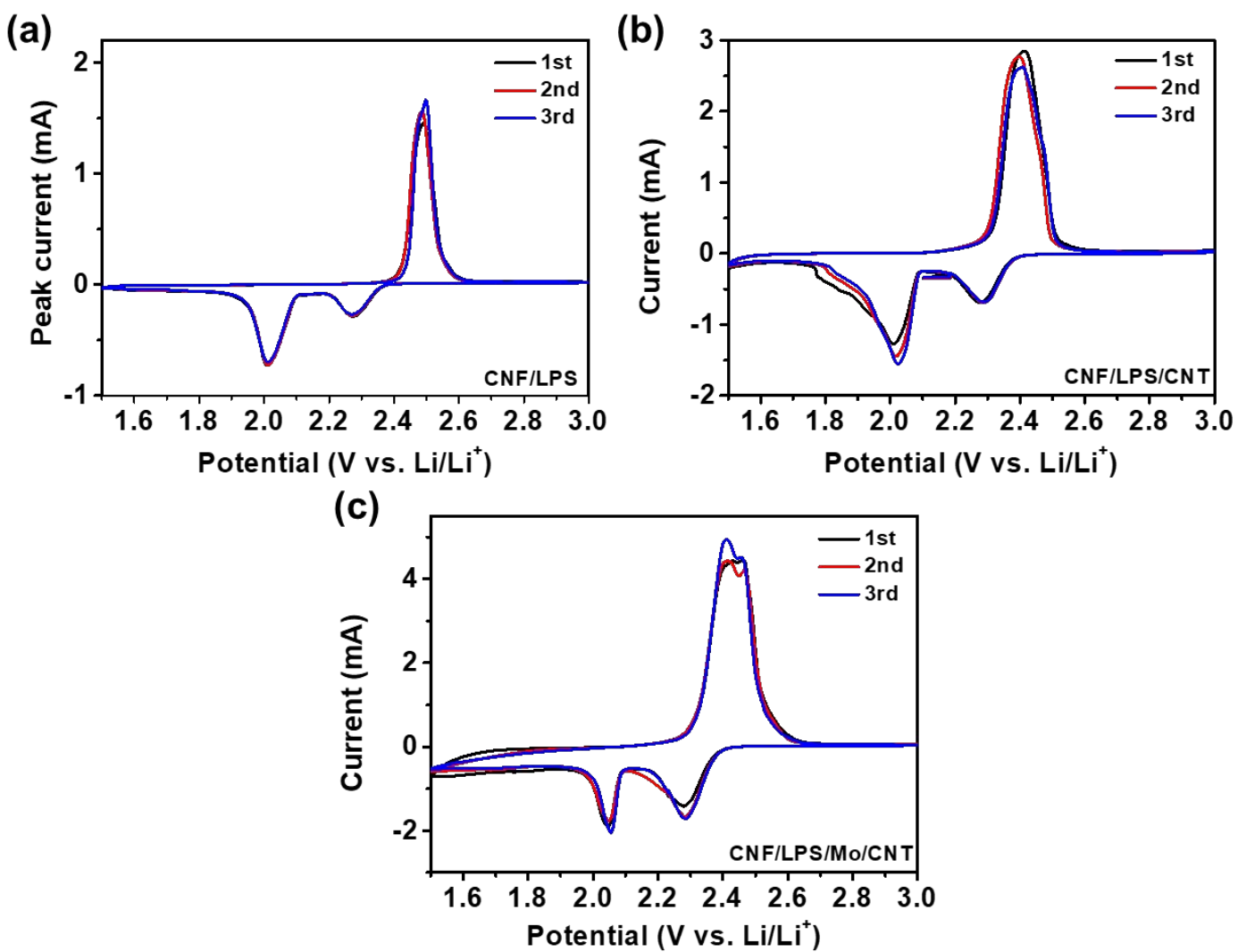

Figure S11. The first-three cycles of CVs for the (a) CNF/LPS, (b) CNF/LPS/CNT, and (c) $\mathrm{CNF} / \mathrm{LPS} / \mathrm{Mo} / \mathrm{CNT}$ cathodes.

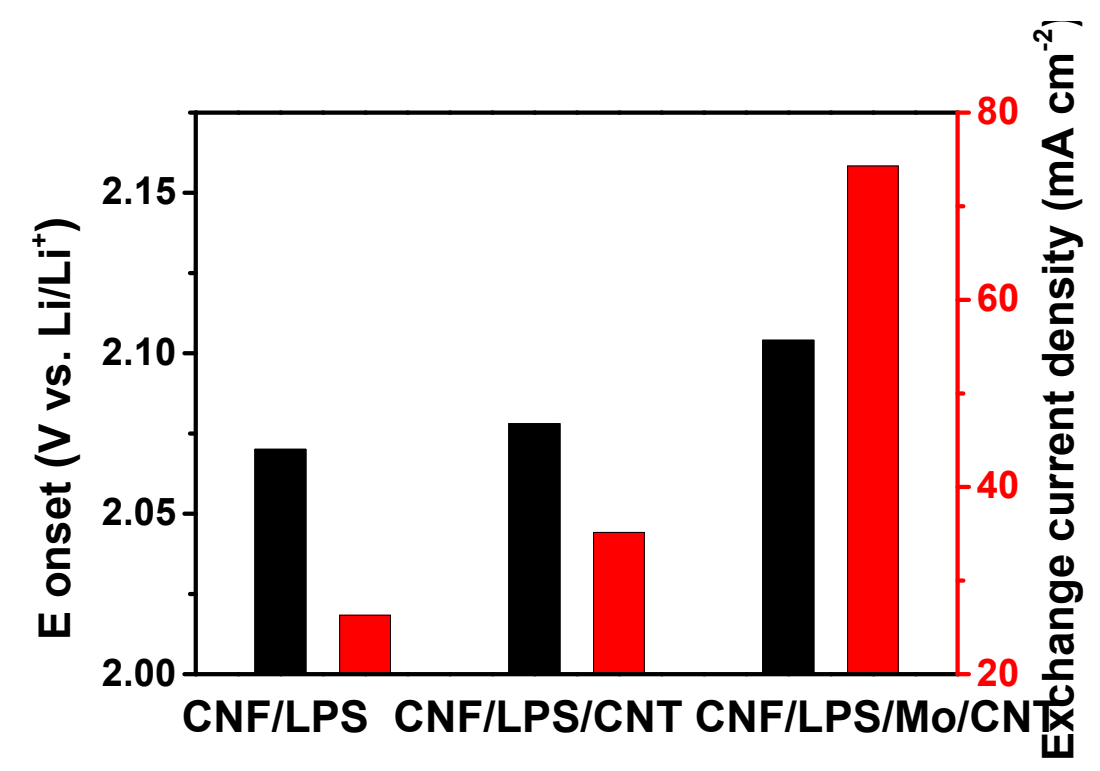

Figure S12. Comparison of the onset potentials and the derived exchange current density for liquid-solid reactions from LPS to $\mathrm{Li}_{2} \mathrm{~S}$. 

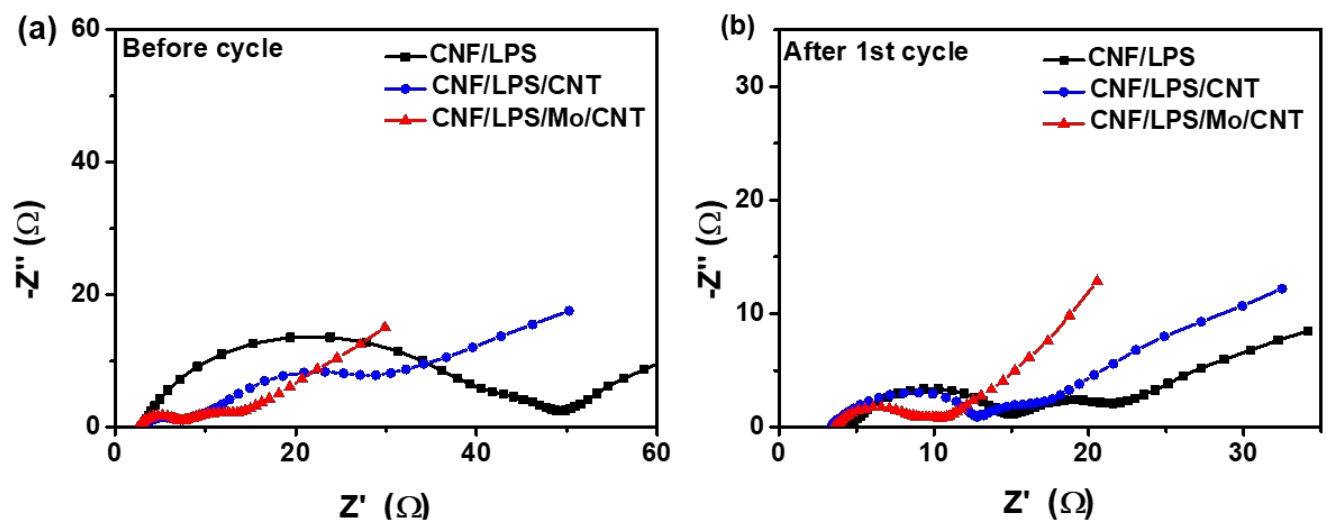

Figure S13. Electrochemical impedance spectra of Li-S cells with CNF/LPS, (b) $\mathrm{CNF} / \mathrm{LPS} / \mathrm{CNT}$, and (c) CNF/LPS/Mo/CNT cathodes (a) before cycling and (b) after the 1 st charge/discharge cycle.

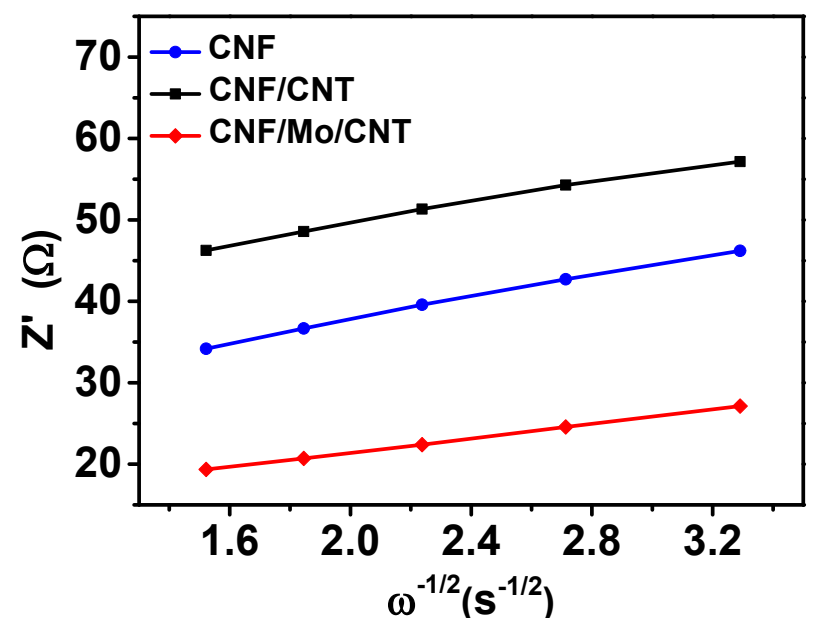

Figure S14. The low-frequency relationships between Zre and $\omega^{1 / 2}$ of CNF/LPS, $\mathrm{CNF} / \mathrm{LPS} / \mathrm{CNT}$, and CNF/LPS/Mo/CNT cathodes.

The Li-ion diffusion coefficient $\left(\mathrm{D}_{\mathrm{Li}}{ }^{+}\right)$values were calculated according to the following equations:

$$
\begin{aligned}
& \mathrm{D}_{\mathrm{Li}}{ }^{+}=\mathrm{R}^{2} \mathrm{~T}^{2} /\left(2 \mathrm{n}^{4} \mathrm{~A}^{2} \mathrm{~F}^{4} \mathrm{C}^{2} \sigma^{2}\right) \\
& \mathrm{Z}_{\mathrm{re}}=\mathrm{R}_{\mathrm{e}}+\mathrm{R}_{\mathrm{ct}}+\sigma \omega^{-1 / 2}
\end{aligned}
$$

Here, $\mathrm{R}$ is the gas constant, $\mathrm{T}$ is the room temperature, $\mathrm{n}$ is electron number for each molecule during $\mathrm{Li}^{+}$insertion, $\mathrm{A}$ is the surface area of the electrode, $\mathrm{F}$ is the Faraday's constant, $\mathrm{C}$ is $\mathrm{Li}^{+}$concentration, $\sigma$ is the Warburg factor which has the relationship with $Z_{\text {re }}$, and $\omega$ is the angular frequency in the low frequency range. As shown in Figure S9, the low-frequency relationships between $Z_{\mathrm{re}}$ and $\omega^{-1 / 2}$ of CNF/LPS, CNF/LPS/CNT, and $\mathrm{CNF} / \mathrm{LPS} / \mathrm{Mo} / \mathrm{CNT}$ were obtained from the EIS spectrum, respectively. The three $\sigma$ values, the slopes of the three fitted Lines, are $6.196,6.792,4.418 \Omega \mathrm{cm}^{2} \mathrm{~s}^{-1 / 2}$, giving the $\mathrm{D}$ values of $9.382 \times 10^{-13}, 4.233 \times 10^{-13}$, and $1.845 \times 10^{-12} \mathrm{~cm}^{2} \mathrm{~s}^{-1}$ for CNF/LPS, $\mathrm{CNF} / \mathrm{LPS} / \mathrm{CNT}$, and CNF/LPS/Mo/CNT, respectively. Since the CNT and CNT/Mo cap layers have same network structure (Figure S3), the promoted Li ions diffusion on $\mathrm{CNF} / \mathrm{LPS} / \mathrm{Mo} / \mathrm{CNT}$ electrode originates suggests the effectiveness of Mo nanocrystal in promoting the fast kinetic of $\mathrm{Li}$ ions and catalytic activation of polysulfides ions. 

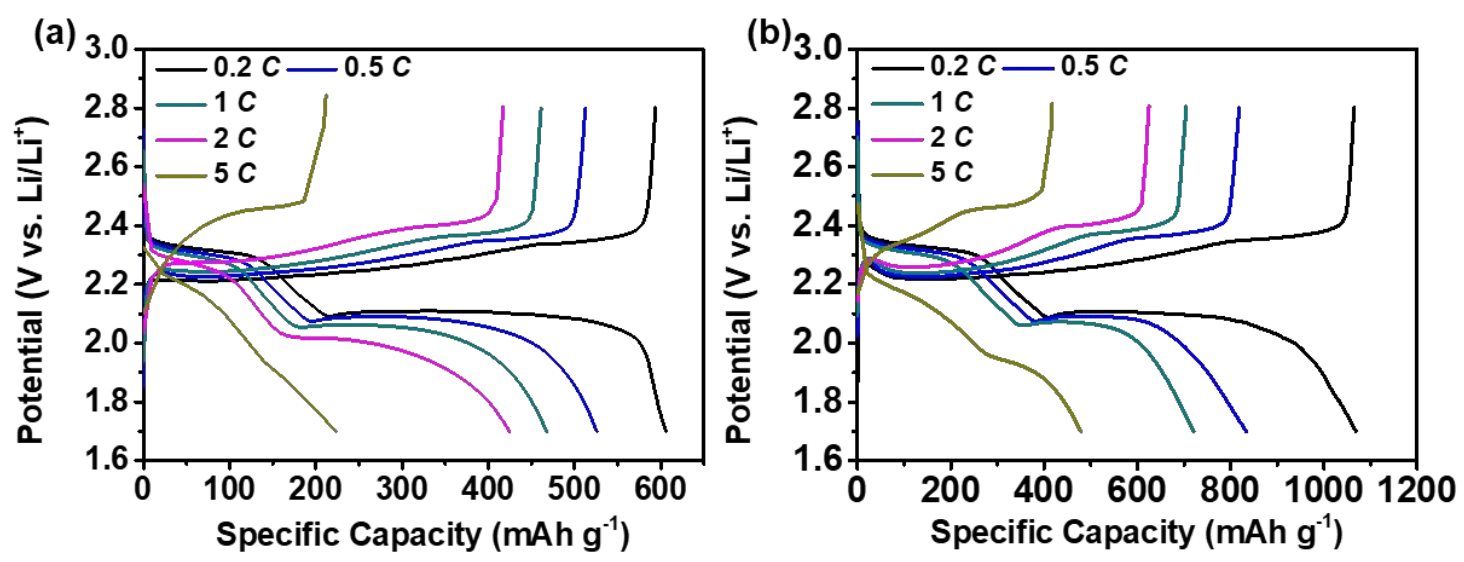

Figure S15. Typical galvanostatic charge-discharge profiles of the (a) CNF/LPS and (b) $\mathrm{CNF} / \mathrm{LPS} / \mathrm{CNT}$ cathodes at $0.2,0.5,1,2$, and $5 C$, respectively.
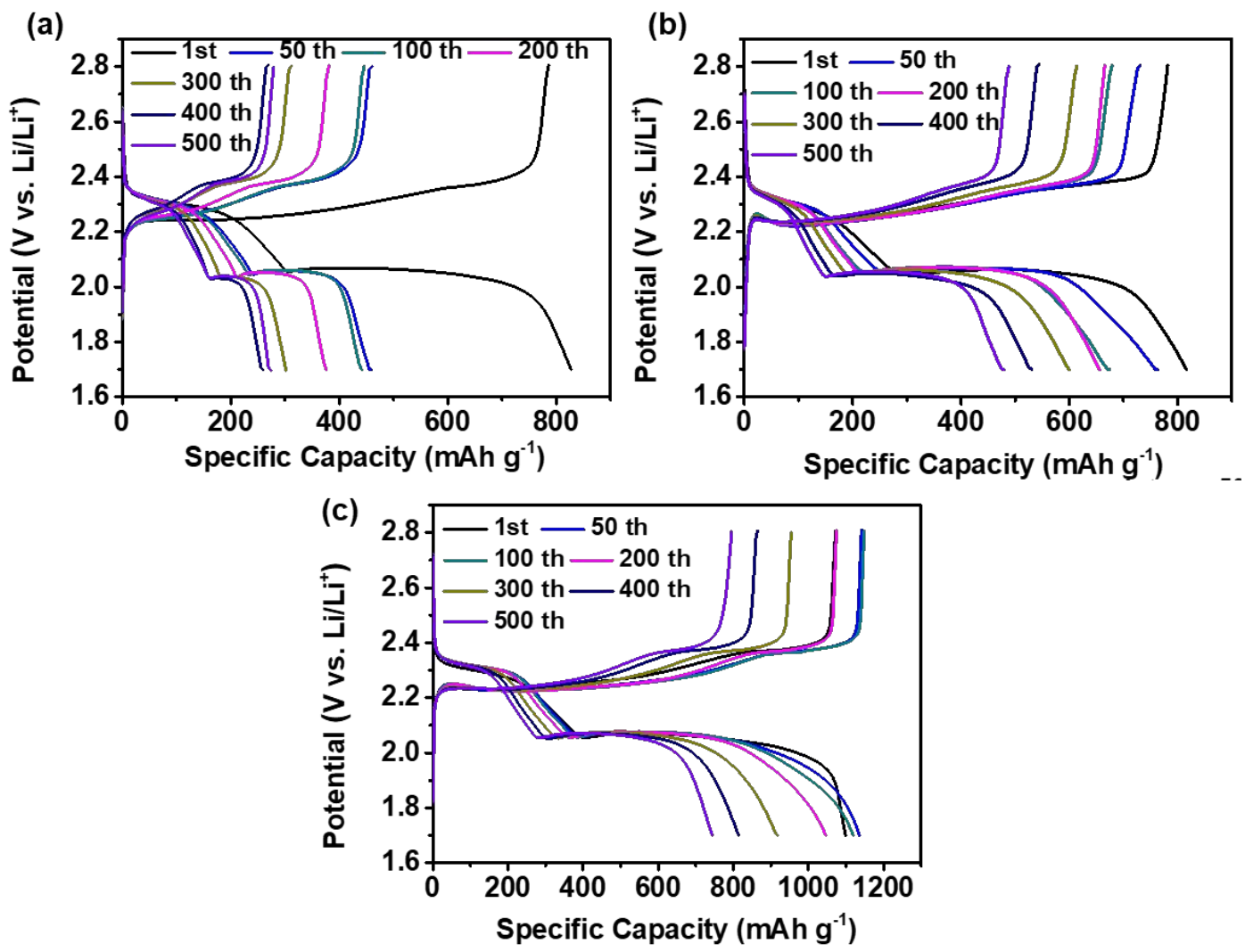

Figure S16. Typical galvanostatic charge-discharge profiles of the (a) CNF/LPS, (b) $\mathrm{CNF} / \mathrm{LPS} / \mathrm{CNT}$, and (c) CNF/LPS/Mo/CNT cathodes for 1st, 50th, 100th, 200th, 300th, 400th, and 500th cycle at $1 C$, respectively. 

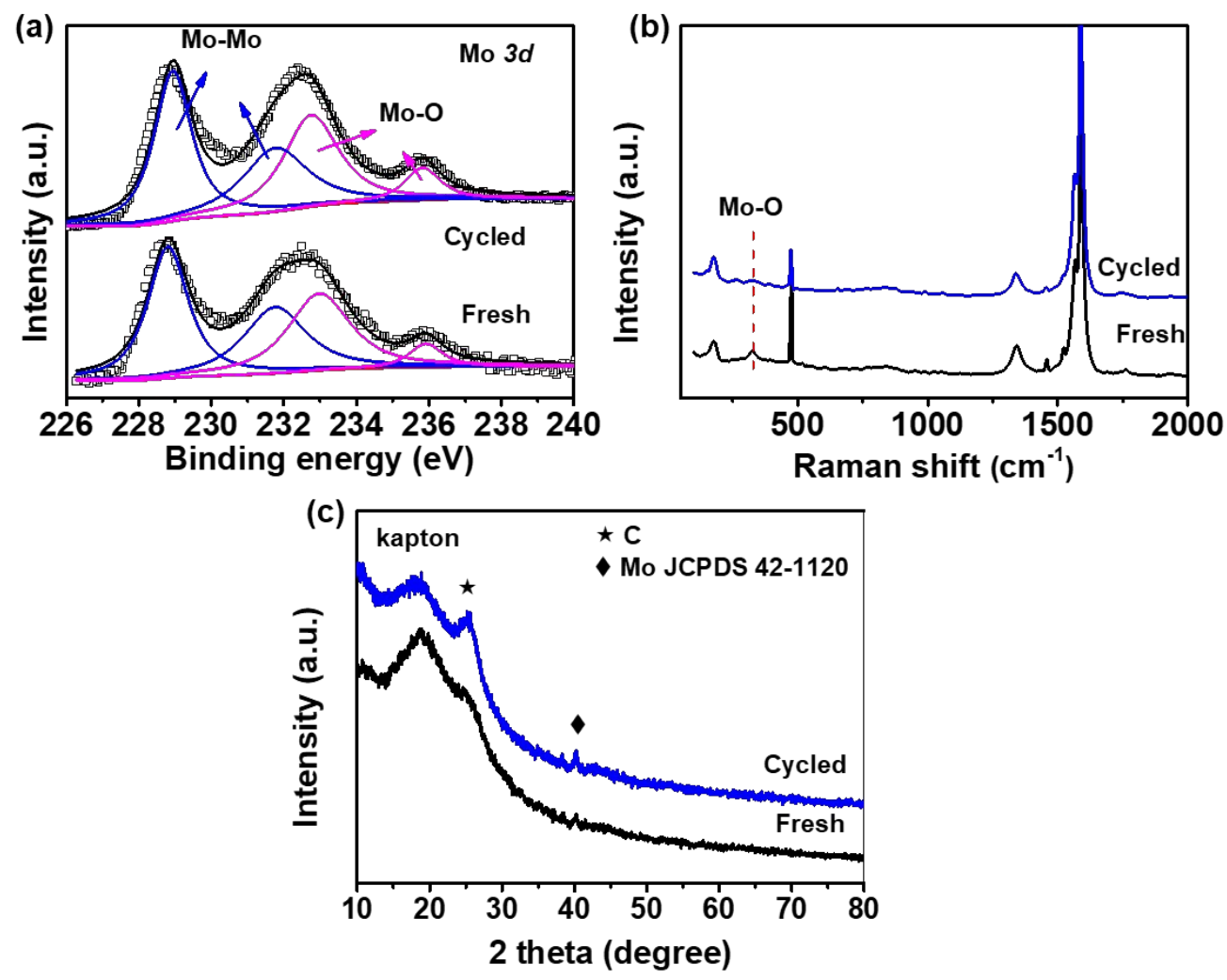

Figure S17. (a) Mo 3d XPS spectra, (b) Raman spectra, and (c) XRD patterns of the CNT/Mo composites before and after 100 cycles at $0.5 C$.
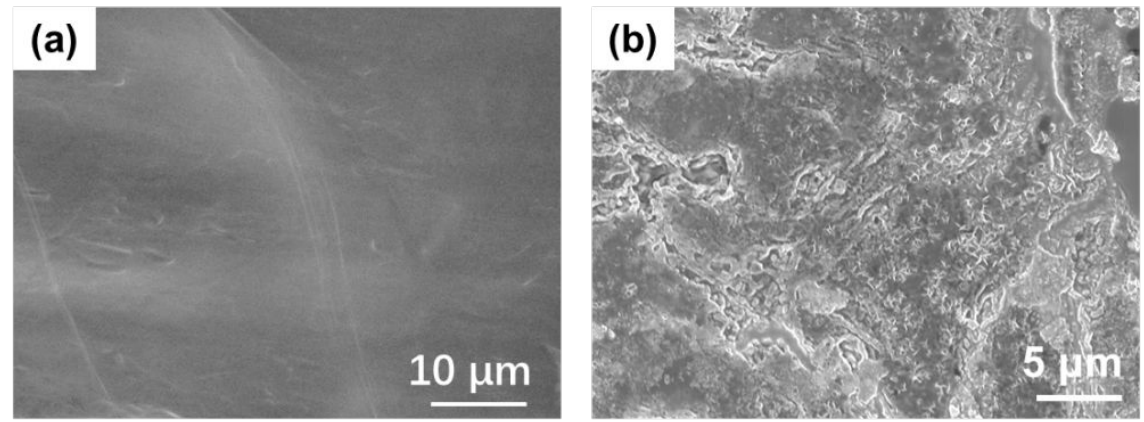

(c)

(d)
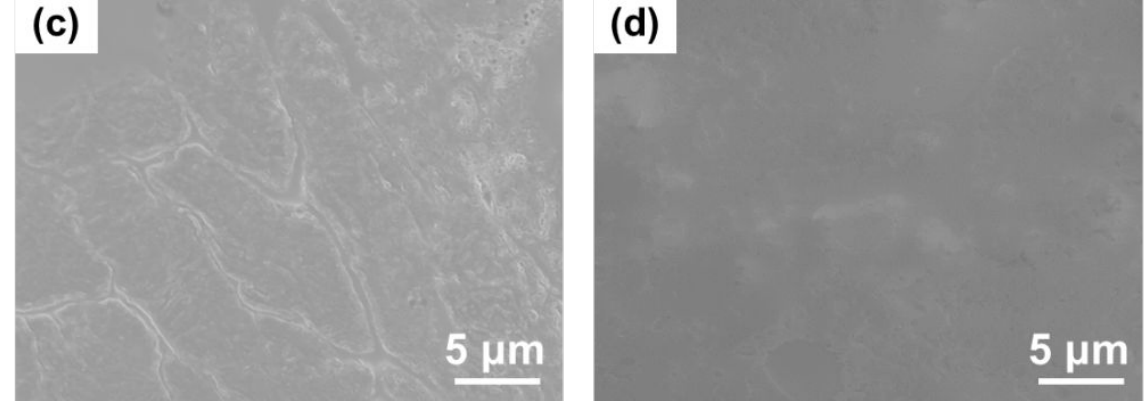

Figure S18. SEM images of Li metal anodes (a) before cycling and after 100 cycles paired with (b) CNF/LPS, (c) CNF/LPS/CNT, and (d) CNF/LPS/Mo/CNT cathodes, respectively. 


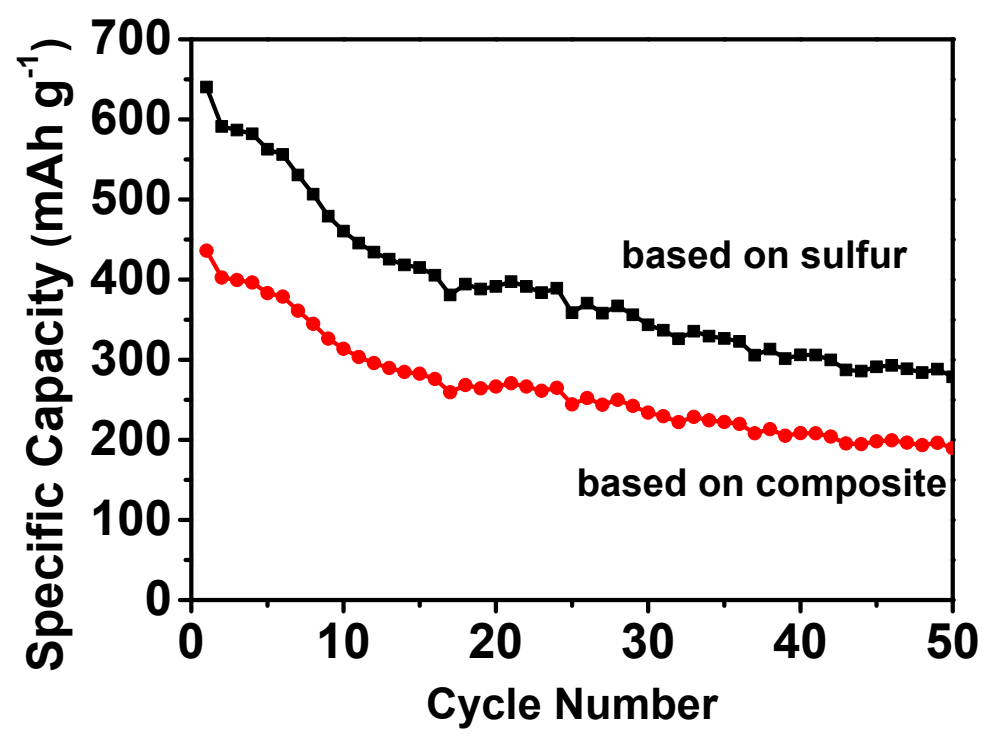

Figure S19. Cycling performance of the CNF/LPS cathode with high sulfur mass loading of $5.73 \mathrm{mg} \mathrm{cm}^{-2}$ at $0.2 C$.
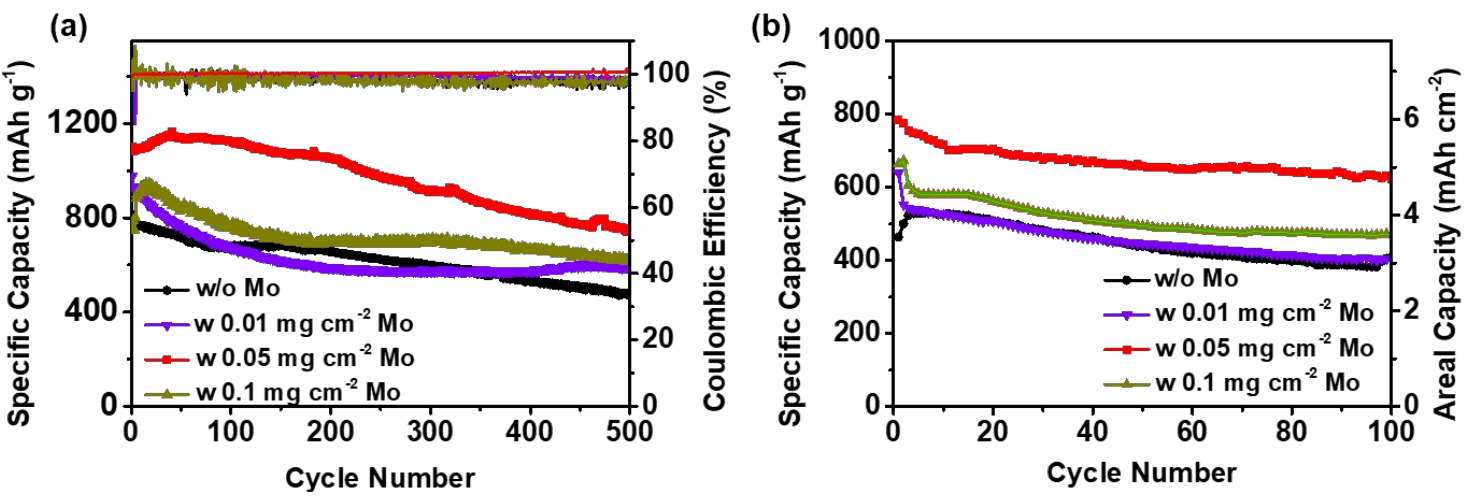

Figure S20. Cycling performance of $\mathrm{CNF} / \mathrm{LPS} / \mathrm{Mo} / \mathrm{CNT}$ cathodes with different loading of metallic Mo layer (a) with sulfur mass loading of $0.96 \mathrm{mg} \mathrm{cm}^{-2}$ at $1 \mathrm{C}$ and (b) with high sulfur mass loading of $7.64 \mathrm{mg} \mathrm{cm}^{-2}$ at $0.2 C$.

(a)

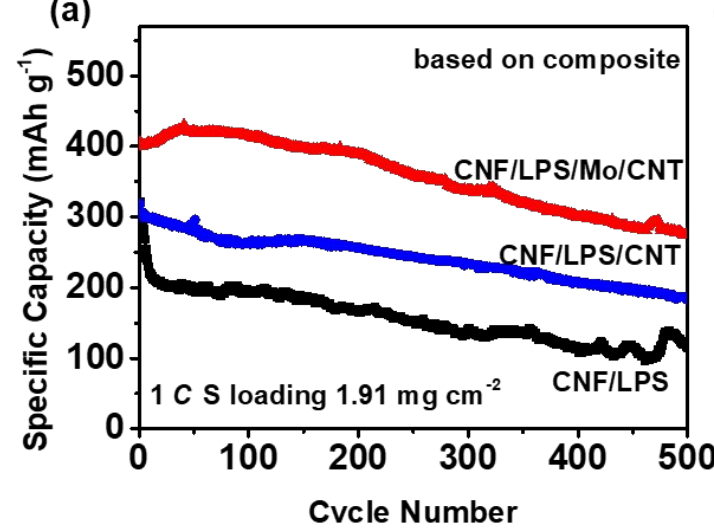

(b)

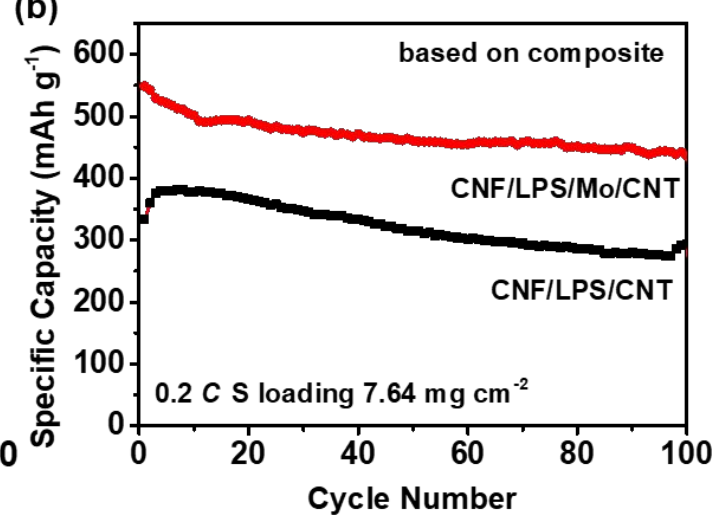

Figure S21. Capacity-cycle number plots of CNF/LPS/Mo/CNT cathodes based on the mass of all the components of the electrodes. 
Table S1. Comparison of electrochemical performance of the CNF/LPS/Mo/CNT cathode with the recent reported sulfur-based cathodes.

\begin{tabular}{|c|c|c|c|c|c|c|}
\hline $\begin{array}{l}\text { Cathode } \\
\text { materials }\end{array}$ & $\begin{array}{c}\text { Concentration } \\
\text { of the } \\
\text { catholyte }\end{array}$ & $\begin{array}{l}\text { Content of } \\
\text { polar } \\
\text { materials in } \\
\text { host }(\mathrm{wt} \%)\end{array}$ & $\begin{array}{c}\text { Sulfur } \\
\text { mass } \\
\text { loading } \\
\left(\mathrm{mg} \mathrm{cm}^{-2}\right)\end{array}$ & $\begin{array}{l}\text { Rate } \\
(C)\end{array}$ & $\begin{array}{l}\text { Capacity } \\
\left(\mathrm{mAh} \mathrm{g}^{-1}\right)\end{array}$ & Ref \\
\hline $\begin{array}{l}\text { CNF/LPS/ } \\
\text { Mo/CNT }\end{array}$ & (0.5 M) & 5 & 1.9 & $\begin{array}{l}1 \\
5\end{array}$ & $\begin{array}{c}744 \text { (500 cycles) } \\
694 \text { (rate capacity) }\end{array}$ & $\begin{array}{l}\text { This } \\
\text { work }\end{array}$ \\
\hline $\begin{array}{l}\mathrm{SnS}_{2} \mathrm{ND} @ \mathrm{G} \\
\text { @ } \mathrm{Li}_{2} \mathrm{~S}_{6}\end{array}$ & $(0.8 \mathrm{M})$ & 16 & 2.5 & $\begin{array}{c}0.2 \\
3\end{array}$ & $\begin{array}{c}1016 \text { (300 cycles) } \\
350 \text { (rate capacity) }\end{array}$ & [S5] \\
\hline $\begin{array}{c}\text { 3DNG/TiN } \\
\text { @ } \mathrm{Li}_{2} \mathrm{~S}_{6}\end{array}$ & $(1.0 \mathrm{M})$ & 31.5 & 4.8 & $\begin{array}{c}0.5 \\
5\end{array}$ & $\begin{array}{l}1265 \text { (100 cycles) } \\
676 \text { (rate capacity) }\end{array}$ & [S6] \\
\hline $\begin{array}{c}\mathrm{Ti}_{3} \mathrm{C}_{2} \mathrm{~T}_{\mathrm{x}} / \\
\mathrm{rGO} @ \mathrm{Li}_{2} \mathrm{~S}_{6} \\
\end{array}$ & $(1.0 \mathrm{M})$ & 30 & 1.57 & $\begin{array}{l}1 \\
1\end{array}$ & $\begin{array}{c}596(500 \text { cycles) } \\
475 \text { (rate capacity) }\end{array}$ & [S7] \\
\hline $\begin{array}{l}\text { VN/Graphe } \\
\text { ne@ } \mathrm{Li}_{2} \mathrm{~S}_{6}\end{array}$ & $(0.33 \mathrm{M})$ & 30 & 1.92 & $\begin{array}{l}1 \\
3\end{array}$ & $\begin{array}{c}917 \text { (200 cycles) } \\
701 \text { (rate capacity) }\end{array}$ & [S8] \\
\hline $\begin{array}{l}\text { FLP-CNF } \\
@ \mathrm{Li}_{2} \mathrm{~S}_{6} \\
\end{array}$ & $(1.0 \mathrm{M})$ & 15 & 2 & $\begin{array}{l}1 \\
3 \\
\end{array}$ & $\begin{array}{c}660 \text { (500 cycles) } \\
785 \text { (rate capacity) }\end{array}$ & [S9] \\
\hline $\begin{array}{c}\mathrm{TiO}_{2} \mathrm{NW} / \mathrm{G} \\
\text { @ } \mathrm{Li}_{2} \mathrm{~S}_{6}\end{array}$ & $(0.5 \mathrm{M})$ & 15 & 3.2 & $\begin{array}{c}0.2 \\
2\end{array}$ & $\begin{array}{l}1053 \text { (200 cycles) } \\
850 \text { (rate capacity) }\end{array}$ & [S10] \\
\hline $\begin{array}{c}\mathrm{W}_{2} \mathrm{C} \text { NPs- } \\
\mathrm{CNFs} @ \\
\mathrm{Li}_{2} \mathrm{~S}_{6}\end{array}$ & $(0.625 \mathrm{M})$ & 59.4 & 2 & $\begin{array}{l}1 \\
2\end{array}$ & $\begin{array}{c}605 \text { (500 cycles) } \\
646 \text { (rate capacity) }\end{array}$ & [S11] \\
\hline
\end{tabular}


Table S2. Comparison of areal capacity of the CNF/LPS/Mo/CNT cathode with the recent reported sulfur-based cathodes with sulfur mass loading higher than $6 \mathrm{mg} \mathrm{cm}^{-2}$.

\begin{tabular}{|c|c|c|c|c|}
\hline $\begin{array}{l}\text { Cathode } \\
\text { materials }\end{array}$ & $\begin{array}{c}\text { Mass } \\
\text { loading } \\
\left(\mathrm{mg} \mathrm{cm}^{-2}\right)\end{array}$ & $\begin{array}{l}\text { Initial areal } \\
\text { capacity } \\
\left.(\mathrm{mAh} \mathrm{cm})^{-2}\right)\end{array}$ & Capacity retention & Ref \\
\hline CNF/LPS/Mo/CNT & 7.64 & 5.98 & $\begin{array}{c}80 \% \\
\text { (after } 100 \text { cycles) }\end{array}$ & $\begin{array}{l}\text { This } \\
\text { work }\end{array}$ \\
\hline $\begin{array}{c}\mathrm{SnS}_{2}-\mathrm{ND} @ \mathrm{G} \\
@ \mathrm{Li}_{2} \mathrm{~S}_{6}\end{array}$ & 10 & 11.3 & $\begin{array}{c}66 \% \\
\text { (after } 100 \text { cycles) }\end{array}$ & {$[\mathrm{S} 5]$} \\
\hline $\begin{array}{c}3 \mathrm{DNG} / \mathrm{TiN} \\
@ \mathrm{Li}_{2} \mathrm{~S}_{6}\end{array}$ & 9.6 & 12.0 & $\begin{array}{c}80 \% \\
\text { (after } 60 \text { cycles) }\end{array}$ & {$[\mathrm{S} 6]$} \\
\hline $\begin{array}{c}\text { N,S-codoped } \\
\text { graphene } @, \mathrm{Li}_{2} \mathrm{~S}_{6}\end{array}$ & 8.5 & 7.86 & $\begin{array}{c}72.4 \% \\
\text { (after } 200 \text { cycles) }\end{array}$ & {$[\mathrm{S} 12]$} \\
\hline $\begin{array}{c}\text { N, S co-doped CP } \\
\text { @ } \mathrm{Li}_{2} \mathrm{~S}_{6}-\mathrm{CMK} 3\end{array}$ & 9 & 9.1 & $\begin{array}{c}78 \% \\
\text { (after } 300 \text { cycles) }\end{array}$ & [S13] \\
\hline CNT sheet@ $\mathrm{Li}_{2} \mathrm{~S}_{6}$ & 10 & 7.8 & $\begin{array}{c}78 \% \\
\text { (after } 300 \text { cycles) }\end{array}$ & [S14] \\
\hline CNF Sheets@ $\mathrm{Li}_{2} \mathrm{~S}_{6}$ & 18.1 & 14.95 & $\begin{array}{c}81 \% \\
\text { (after } 75 \text { cycles) }\end{array}$ & [S15] \\
\hline 3D GF-rGO/S & 9.8 & 8.5 & $\begin{array}{c}74 \% \\
\text { (after } 350 \text { cycles) }\end{array}$ & {$[\mathrm{S} 16]$} \\
\hline $\begin{array}{c}\text { Free-Standing CNT-S } \\
\text { Paper }\end{array}$ & 6.3 & 6.2 & $\begin{array}{c}71 \% \\
\text { (after } 150 \text { cycles) }\end{array}$ & {$[\mathrm{S} 17]$} \\
\hline $\begin{array}{c}\text { S-PDMS/Graphene } \\
\text { Foam }\end{array}$ & 10.1 & 9.3 & $\begin{array}{c}48.6 \% \\
\text { (after } 1000 \text { cycles) }\end{array}$ & {$[\mathrm{S} 18]$} \\
\hline
\end{tabular}


Table S3. Design parameters for calculations of cell specific energy density.

\begin{tabular}{|c|c|}
\hline \multicolumn{2}{|c|}{ Design parameters for calculations of cell specific energy density } \\
\hline Cell part & Weight of material for $\mathrm{Li}-\mathrm{S}$ cell $(\mathrm{mg})$ \\
\hline $\mathrm{Li}$ electrode $(150 \%$ excess) & 3 \\
\hline Electrolyte & 20 \\
\hline Separator & 2.55 \\
\hline CNF+CNT+Mo & $1.2+0.3+0.14$ \\
\hline Catholyte (LPS + solvent) & $3.84+40$ \\
\hline
\end{tabular}

The gravimetric energy density was calculated from $\mathrm{S} 19, \mathrm{~S} 20$ :

$W_{\text {cell }}=E_{\text {cell }} * Q * m_{s} / M_{\text {cathode }}=2.1 \mathrm{~V} * 782.7 \mathrm{mAh} \mathrm{g}^{-1 * 3.84 \mathrm{mg} /(3.84+40+1.2+0.3+0.14)}$ $\mathrm{mg}=138.8 \mathrm{Wh} \mathrm{Kg}^{-1}$

Where $W_{\text {cell }}$ is the energy density $\left(\mathrm{Wh} \mathrm{Kg}^{-1}\right), E_{\text {cell }}(\mathrm{V})$ is the average reversible potential (2.1 V vs. $\left.\mathrm{Li} / \mathrm{Li}^{+}\right), Q$ is the specific capacity of sulfur $\left(782.7 \mathrm{mAh} \mathrm{g}^{-1}\right)$, and $M_{\text {cathode }}$ (mg) is the total mass of the CNF/LPS/Mo/CNT cathode and solvent that dissolves the polysulfide species. 
Table S4. Comparison of gravimetric energy density of the CNF/LPS/Mo/CNT cathode with the recent reported sulfur-based cathodes.

\begin{tabular}{|c|c|c|c|c|c|}
\hline Cathode & $\begin{array}{c}\text { Sulfur } \\
\text { loading } \\
\left(\mathrm{mg} \mathrm{cm}^{-2}\right)\end{array}$ & $\begin{array}{c}\text { E/S } \\
\text { ration } \\
\left(\mathrm{mL} \mathrm{g}^{-1}\right)\end{array}$ & $\begin{array}{c}\text { Initial areal } \\
\text { capacity } \\
\left(\mathrm{mAh} \mathrm{cm}^{-2}\right)\end{array}$ & $\begin{array}{c}\text { Gravimetric } \\
\text { energy density } \\
\left(\mathrm{Wh} \mathrm{kg}^{-1}\right)\end{array}$ & Ref \\
\hline $\begin{array}{c}\text { CNF/LPS/Mo/ } \\
\text { CNT }\end{array}$ & $\mathbf{7 . 6 4}$ & $\mathbf{1 5 . 6}$ & $\mathbf{5 . 9 8}$ & $\mathbf{1 3 8 . 8}$ & $\begin{array}{c}\text { This } \\
\text { work }\end{array}$ \\
\hline S/PCNF/CNT & 12 & 20 & 13.5 & 109.7 & {$[\mathrm{~S} 21]$} \\
\hline S/CNT/NFC & 8.1 & 30 & 8 & 65.7 & {$[\mathrm{~S} 22]$} \\
\hline S@NCFF & 3 & 20 & 2.4 & 76.7 & {$[\mathrm{~S} 23]$} \\
\hline SN-HCS/S & 2.5 & 32 & 3.5 & 89.2 & {$[\mathrm{~S} 24]$} \\
\hline S@CNF/rGO & 20.3 & 15.6 & 15.5 & 93.4 & {$[\mathrm{~S} 25]$} \\
\hline NCF-S@rGO & 8.8 & 9 & 6.6 & 150.9 & {$[\mathrm{~S} 26]$} \\
\hline SWCNT & 16 & 9.5 & 12.3 & 96.9 & {$[\mathrm{~S} 27]$} \\
\hline /SNF-S & 8.1 & 15 & 10.61 & 127.4 & {$[\mathrm{~S} 28]$} \\
\hline PCF/VN/S & & & & & \\
\hline
\end{tabular}

\section{Supplementary references}

[S1]Dieterle, M.; Mestl, G. Raman Spectroscopy of Molybdenum Oxides Part II. Resonance Raman Spectroscopic Characterization of the Molybdenum Oxides $\mathrm{Mo}_{4} \mathrm{O}_{11}$ and $\mathrm{MoO}_{2}$. Phys. Chem. Chem. Phys. 2002, 4, 822-826.

[S2] Huang, S. Z.; Lim, Y. V.; Zhang, X. M.; Wang, Y.; Zheng, Y.; Kong, D. Z.; Ding, M.; Yang S. A.; Yang, H. Y. Regulating the Polysulfide Redox Conversion by Iron Phosphide Nanocrystals for High-Rate and Ultrastable Lithium-Sulfur Battery. Nano Energy, 2018, 51, 340-348.

[S3] Vinayan, B. P.; Diemant, T.; Lin, X. M.; Cambaz, M. A.; Golla-Schindler, U.; Kaiser, U.; Behm R. J.; Fichtner, M. Nitrogen Rich Hierarchically Organized Porous Carbon/Sulfur Composite Cathode Electrode for High Performance Li/S Battery: A Mechanistic Investigation by Operando Spectroscopic Studies. Adv. Mater. Interfaces, 2016, 3, 1600372 .

[S4] Sato, S.; Higuchi, S.; Tanaka, S. Identification and Determination of OxygenContaining Inorganosulfur Compounds by Laser Raman Spectrometry. Appl. Spectrosc. 1985, 39, 822-827. 
[S5] L. Luo, S.-H. Chung, A. Manthiram, A. Three-Dimensional Self-Assembled $\mathrm{SnS}_{2}-$ Nano-Dots@Graphene Hybrid Aerogel as an Efficient Polysulfide Reservoir for HighPerformance Lithium-Sulfur Batteries. J. Mater. Chem. A 2018, 6, 7659-7667.

[S6] Li, Z.; He, Q.; Xu, X.; Zhao, Y.; Liu, X.; Zhou, C.; Ai, D.; Xia, L.; Mai, L. A 3D Nitrogen-Doped Graphene/TiN Nanowires Composite as a Strong Polysulfide Anchor for Lithium-Sulfur Batteries with Enhanced Rate Performance and High Areal Capacity Adv. Mater. 2018, 30, 1804089.

[S7] Song, J.; Guo, X.; Zhang, J.; Chen, Y.; Zhang, C.; Luo, L.; Wang, F.; Wang, G. Rational Design of Free-standing 3D Porous MXene/rGO Hybrid Aerogels as Polysulfide Reservoirs for High-Energy Lithium-Sulfur Batteries. J. Mater. Chem. A 2019, 7, 6507 .

[S8] Sun, Z.; Zhang, J.; Yin, L.; Hu, G.; Fang, R.; Cheng, H.-M.; Li, F. Conductive Porous Vanadium Nitride/Graphene Composite as Chemical Anchor of Polysulfides for Lithium-Sulfur Batteries. Nat. Commun. 2017, 8, 14627.

[S9] Li, L.; Chen, L.; Mukherjee, S.; Gao, J.; Sun, H.; Liu, Z.; Ma, X.; Gupta, T.; Singh, C. V.; Ren, W.; Cheng, H.-M.; Koratkar N. Phosphorene as a Polysulfide Immobilizer and Catalyst in High-Performance Lithium-Sulfur Batteries. Adv. Mater. 2017, 29, 1602734.

[S10] Zhou, G.; Zhao, Y.; Zu, C.; Manthiram, A.; Free-standing $\mathrm{TiO}_{2}$ NanowireEmbedded Graphene Hybrid Membrane for Advanced Li/Dissolved Polysulfide Batteries. Nano Energy 2015, 12, 240-249.

[S11] Zhou, F.; Li, Z.; Luo, X.; Wu, T.; Jiang, B.; Lu, L.-L.; Yao, H.-B.; Antonietti, M.; Yu, S.-H. Low Cost Metal Carbide Nanocrystals as Binding and Electrocatalytic Sites for High Performance Li-S Batteries. Nano Lett. 2018, 18, 1035-1043.

[S12] Zhou, G.; Paek, E.; Hwang, G. S.; Manthiram, A. Long-life Li/Polysulphide Batteries with High Sulphur Loading Enabled by Lightweight Three-Dimensional Nitrogen/Sulphur-Codoped Graphene Sponge. Nat. Commun. 2015, 6, 7760.

[S13] Wang, X.; Gao, T.; Han, F.; Ma, Z.; Zhang, Z.; Li, J.; Wang, C. Stabilizing High Sulfur Loading Li-S Batteries by Chemisorption of Polysulfide on Three-Dimensional Current Collector. Nano Energy 2016, 30, 700-708. 
[S14] Pan, Z.-Z. ; Lv, W.; He, Y.-B.; Zhao, Y.; Zhou, G.; Dong, L.; Niu, S.; Zhang, C.; Lyu, R.; Wang, C.; Shi, H.; Zhang, W.; Kang, F.; Nishihara, H.; Yang, Q.-H. A NacreLike Carbon Nanotube Sheet for High Performance Li-Polysulfde Batteries with High Sulfur Loading. Adv. Sci. 2018, 5, 1800384.

[S15] Qie, L.; Zu, C.; Manthiram, A High Energy Lithium-Sulfur Battery with Ultrahigh-Loading Lithium Polysulfide Cathode and its Failure Mechanism. $A d v$. Energy Mater. 2016, 6, 1502459.

[S16] Hu, G.; Xu, C.; Sun, Z.; Wang, S.; Cheng, H.-M.; Li, F.; Ren, W. 3D GrapheneFoam-Reduced-Graphene-Oxide Hybrid Nested Hierarchical Networks for HighPerformance Li-S Batteries. Adv. Mater. 2016, 28, 1603-1609.

[S17] Yuan, Z.; Peng, H.-J.; Huang, J.-Q.; Liu, X.-Y.; Wang, D.-W.; Cheng, X.-B.; Zhang, Q. Hierarchical Free-Standing Carbon-Nanotube Paper Electrodes with Ultrahigh Sulfur-Loading for Lithium-Sulfur Batteries. Adv. Funct. Mater. 2014, 24, 6105-6112.

[S18] Zhou, G.; Li, L.; Ma, C.; Wang, S.; Shi, Y.; Koratkar, N.; Ren, W.; Li, F.; Cheng, H.-M. A Graphene Foam Electrode with High Sulfur Loading for Flexible and High Energy Li-S Batteries. Nano Energy 2015, 11, 356-365.

[S19] Cha, E.; Patel, M. D.; Park, J.; Hwang, J.; Prasad, V.; Cho, K.; Choi, K. 2D MoS 2 as an Efficient Protective Layer for Lithium Metal Anodes in High-Performance Li-S Batteries. Nat. Nanotechnol. 2018, 13, 337-344.

[S20] Wang, L.; Liu, J.; Haller, S.; Wang, Y.; Xia, Y. A Scalable Hybrid Separator for a High-Performance Lithium-Sulfur Battery. Chem. Commun. 2015, 51, 6996.

[S21] Zhang, Y. Z.; Zhang, Z.; Liu, S.; Li, G. R.; Gao, X. P. Free-Standing Porous Carbon Nanofiber/Carbon Nanotube Film as Sulfur Immobilizer with High Areal Capacity for Lithium-Sulfur Battery. ACS Appl. Mater. Interfaces 2018, 10, 8749-8757. [S22] Yu, M.; Ma, J.; Xie, M.; Song, H.; Tian, F.; Xu, S.; Zhou, Y.; Li, B.; Wu, D.; Qiu, H.; Wang, R. Freestanding and Sandwich-Structured Electrode Material with High Areal Mass Loading for Long-Life Lithium-Sulfur Batteries. Adv. Energy Mater. 2017, 7,1602347 
[S23] Ren, W.; Ma, W.; Zhang, S.; Tang, B. Nitrogen-Doped Carbon Fiber Foam Enabled Sulfur Vapor Deposited Cathode for High Performance Lithium Sulfur Batteries. Chem. Eng. J. 2018, 341, 441-449.

[S24] Yang, D.; Ni, W.; Cheng, J.; Wang, Z.; Wang, T.; Guan, Q.; Zhang, Y.; Wu, H.; Li, X.; Wang, B. Flexible Three-Dimensional Electrodes of Hollow Carbon Bead Strings as Graded Sulfur Reservoirs and the Synergistic Mechanism for Lithium-Sulfur Batteries. Appl. Surf. Sci. 2017, 413, 209-218.

[S25] Han, S.; Pu, X.; Li, X.; Liu, M.; Li, M.; Feng, N.; Dou, S.; Hu, W. High Areal Capacity of Li-S Batteries Enabled by Freestanding CNF/rGO Electrode with High Loading of Lithium Polysulfide. Electrochim. Acta 2017, 241, 406-413.

[26] Xiang, M.; Yang, L.; Zheng, Y.; Huang, J.; Jing, P.; Wu, H.; Zhang, Y.; Liu, H. A Freestanding and Flexible Nitrogen-Doped Carbon Foam/Sulfur Cathode Composited with Reduced Graphene Oxide for High Sulfur Loading Lithium-Sulfur Batteries. $J$. Mater. Chem. A 2017, 5, 18020-18028.

[27] Chang, C.-H.; Chung, S.-H.; Manthiram, A. Highly Flexible, Freestanding Tandem Sulfur Cathodes for Foldable Li-S Batteries with a High Areal Capacity. Mater. Horiz. 2017, 4, 249-258.

[28] Zhong, Y.; Chao, D.; Deng, S.; Zhan, J.; Fang, R.; Xia, Y.; Wang, Y.; Wang, X.; Xia, X.; Tu, J. Confining Sulfur in Integrated Composite Scaffold with Highly Porous Carbon Fibers/Vanadium Nitride Arrays for High-Performance Lithium-Sulfur Batteries. Adv. Funct. Mater. 2018, 28, 1706391. 